\title{
"Y ATRAVESÓ EL MAR INFERIOR": LA DINASTÍA ACADIA Y SU MIRADA A LAS COSTAS DEL GOLFO PÉRSICO
}

\author{
Carmen del Cerro Linares \\ (Universidad Autónoma de Madrid)
}

\begin{abstract}
RESUMEN
La primera mención que tenemos de la región de Magan en los textos Mesopotámicos aparece en época acadia, siempre asociada a las regiones de Dilmun y Meluhha. Mientras que regiones como Dilmun quedan ya consignadas en los textos Uruk o expresiones como "Mar Inferior" aparecen ya a finales del Dinástico Temprano, la región de Magan y lo que los textos acadios llamarán "Montañas Negras" no quedan recogidas hasta época acadia, en concreto desde el reinado de Maništušu. En este artículo vamos a hacer un recorrido por los textos mesopotámicos que nos hablan de Magan en época acadia, por los materiales que provienen de esas montañas (las actuales Montañas de al Hayyar) y por restos arqueológicos de la cultura Umm an Nar, contemporánea al mundo acadio en la Península de Omán. Entre ambos Acad y Magan, la región de Dilmun, intermediaria por excelencia, tendrá también cabida en nuestro estudio, por ser lugar obligatorio de trasvase de los materiales, los objetos y las ideas que circularon por el Golfo Pérsico en la segunda mitad del III milenio a.C.
\end{abstract}

\section{PALABRAS CLAVE}

Dinastía acadia, Dilmun, Magan, Montañas Negras, Umm an Nar, Hili, cobre, diorita, comercio

\section{'AND HE CROSSED THE LOWER SEA': THE AKKADIAN DYNASTY AND ITS LOOK TO THE PERSIAN GULF COASTS}

\begin{abstract}
The first mention which we have on the region of Magan in the Mesopotamian texts appears in the Akkadian Age, always connected to the regions of Dilmum and Meluhha. While regions such as Dilmun, recorded in the Uruk texts or even as an expression like 'Lower Sea' already appear at the end of the Early Dynastic, the region of Magan, what will be called in the texts 'Black Mountains', are not recorded until the Akkadian times, concretely since Maništušu's reign. In this article, we are going to take an historical tour on the Mesopotamian texts which tell us about Magan in the Akkadian Age. We are building this on both the materials coming from those mountains (current mountains of al Hayyar) and the archaeological remains of the Umm an Nar Culture, contemporary of the Akkadian World in Oman Peninsula. Between them, Akkad and Magan, the region of Dilmun, intermediary by excellence, will also have a place in our investigation, as it is a compulsory place of transfer of the materials, objects and the ideas which circulated all over the Persian Gulf in the Second half of the III Millennium BC.
\end{abstract}

\section{KEYWORDS}

Akkadian Dynasty, Dilmun, Magan, Black Mountains, Umm an Nar, Hili, Cupper, Diorite, Trade

\section{Introducción}

Resultaba inquietante, a principios de los años ochenta del siglo pasado, asegurar con rotundidad que los investigadores éramos capaces de situar con exactitud las dos regiones que los textos Mesopotámicos llaman Dilmun y Magan. Pero actualmente, tras décadas de descubrimientos arqueológicos y estudios cronológicos ${ }^{1}$, a nadie le asombrará leer

${ }^{1}$ D.T. POTTS, "The chronology of archaeological assemblages from the head of the Arabian Gulf to the Arabian Sea (8000-1750 BC)" en R. W. Ehrich (ed.), Chronologies in Old World Archaeology, 1992a, Chapter IV, pp. 
que Dilmun ocuparía la parte norte del Golfo Pérsico, es decir: Bahrein, Qatar, el este de la Península Arábiga y quizás la isla de Failaka, mientras que Magan se nos revela en la Península de Omán, el punto más sureste de la Península de Arabia, allí donde los actuales Emiratos Árabes Unidos y el Sultanato de Omán se reparten una porción de tierra vertebrada por las Montañas de al Hayyar o las Montañas de Omán; las atrayentes "Montañas Negras" anotadas en los textos acadios (Fig.1).

Nuestro interés, desde el mismo momento que pusimos rumbo a la Península de Omán por primera vez, ha sido conocer y reconstruir, en la medida de lo posible, la Historia de Magan, pero sin perder nunca de vista el horizonte Mesopotámico en el que nos habíamos formado $^{2}$, por eso nuestro equipo ${ }^{3}$ ha dedicado buena parte de su investigación a atar lazos entre las diferentes entidades que conforman el Golfo en la Antigüedad, y "atravesando el Mar Inferior", intentamos ahora hacer un ejercicio similar al que impulsó la dinastía acadia, que gobernó Mesopotamia entre 2350 y 2300 a.C. aproximadamente.

Las relaciones entre Mesopotamia y el Golfo Pérsico desde fines del IV milenio a.C. quedaron pronto atestiguadas, puesto que tanto cerámica Obeid como Yemdet Nașr fue hallada en Omán y Emiratos Árabes Unidos en las primeras excavaciones danesas en el área ${ }^{4}$ y porque el topónimo Dilmun aparece en los textos mesopotámicos desde el período Uruk Tardío y hasta época Seleucida. Así, la primera mención de ni.tuki ${ }^{\mathbf{k}}$-Dilmun- aparece en las tablillas Uruk, en lo que conocemos como Lista de profesiones donde aparece un "recaudador de impuestos de Dilmun"5.

Pero mientras que Mesopotamia olvidaba a Magan en sus primeros textos, Magan no descuidó a Mesopotamia en las primeras intervenciones arqueológicas que se hicieron en Omán, al menos eso nos dicen las llamadas tumbas Hafit. La cultura Hafit ${ }^{6}$, que se desarrolló en el paso del IV milenio al III a.C., queda manifestada casi exclusivamente en sus tumbas, que se identificaron primero a los pies del yebel Hafit, en al Ain (Abu Dhabi), y fueron prospectadas en primera instancia por P. Glob y excavadas por K. Frifelt ${ }^{7}$ entre 1972/73. Ante unas estructuras casi desconocidas y unos restos óseos muy mal conservados, lo primero que resonó en la expedición danesa fue la existencia de cerámicas muy típicas en época Yemdet Nașr ${ }^{8}$, de importación, junto a producción local; vasijas de forma bicónica, cuello corto, labio exvasado y diseños geométricos insertos en unos paneles en el hombro...formas propias del valle del Diyala y el sur de Mesopotamia a finales del IV milenio a.C. El análisis cerámico no solo nos hablaba de la

63-89; Vid. Figure 1 en D.T. POTTS, "The Late Prehistoric, Protohistoric, and Early Historic Periods in Eastern Arabia (ca. 5000-1200 B.C.)" Journal of World Prehistory 7/2, 1993a, p. 169.

${ }^{2} \mathrm{Y}$ en este punto, en el de la formación histórica y filológica mesopotámica, es donde ancla la autora de este artículo y co-editora de este volumen su gratitud primero, y su cercanía y amistad después, con el Prof. K. Kessler. Como simple estudiante de doctorado, fui acogida por el Prof. K. Kessler en la universidad de Erlangen-Nürnberg donde tuve la fortuna de estudiar acadio con el gran maestro, así como Glíptica Próximo oriental. Sus clases podrían llegar a olvidarse, pero su genio, maestría y agudeza no podrán jamás abandonar ni mis recuerdos ni mi alma.

${ }^{3}$ Nuestro equipo se inserta dentro del grupo de investigación de la UAM Culturas, tecnologías y medio ambiente de las sociedades del Oriente Próximo antiguo dirigido por J.M Córdoba, trabajando en la Península de Omán desde el Proyecto al Madam, Sharjah, EAU.

${ }^{4}$ K. FRIFELT, “A possible link between Jemdet Nasr and the Umm an-Nar graves of Oman” JOS 1, 1975a, passim.

${ }^{5}$ R. ENGLUND, "Dilmun in the Archaic Uruk Corpus" BBVO 2, 1983, p. 35.

${ }^{6}$ M. MAÑÉ, La arqueología de la muerte en la península de Oman (III mil. a.C.) Prácticas y creencias en la región del Piedemonte, Supplementa ad Isimu I. Series: Sudia vol 3, 2005, pp. 50-53 y 65-71.

${ }^{7}$ K. FRIFELT, 1975, passim.

${ }^{8}$ D.T. POTTS, "Eastern Arabia and the Oman peninsula during the late fourth and early third millennium BC" en U. Finkbeiner y W. Röllig (eds.), Gamdat Nasr: Period or Regional Style?, 1986, passim. 
forma sino de una sorprendente realidad, la arcilla con la que se habían realizado los recipientes foráneos de las tumbas Hafit provenía del sur de Mesopotamia, tal y como S. Méry demostraba a principios de los años noventa ${ }^{9}$. ¿Supone esto el establecimiento de comerciantes mesopotámicos a los pies de las Montañas de Cobre -las Montañas Negras de Magan-, siguiendo los parámetros de las famosísimas colonias Uruk en el Éufrates medio a principios del III milenio a.C., cuando el mundo sumerio está apunto de eclosionar? No lo parece, Los túmulos Hafit, que ahora se nos revelan a lo largo de toda la península de Omán, nos parecen enterramientos de población local que ve en las cerámicas de importación mesopotámicas un objeto de valor con el que enterrarse.

A pesar de la evidente y constante relación de las culturas establecidas en todo el Golfo desde el punto de vista de la cultura material, el mundo sumerio continuó sin mencionar la región de Magan. De sus reyes solo Ur Nanše de Lagaš dice, hasta en seis de sus inscripciones, que hace traer los barcos de Dilmun ${ }^{10}$, pero nunca menciona Magan y solo Lugalzagesi, mira hacia el sur de manera global y nos indica que ha puesto orden en el Mar Inferior ${ }^{11}$; la mirada acadia al Golfo Pérsico, contaba con un precursor.

\section{Los textos acadios}

Y así fue. A lo largo de la dinastía acadia podemos ver como nuestros dos mundos se reconocen y nos lo hacen saber. Por primera vez Mesopotamia deja constancia escrita de su interés por el Mar Inferior y las regiones situadas en sus orillas. El primer monarca acadio que habla de Magan es Sargon de Acad (2334-2279 a.C.), tal y como leemos en una de sus inscripciones reales bilingües ${ }^{12}$ (sumerio-acadio), líneas 1-13:

\section{1 [sár-um-gi] / 2. [lugal] / 3. [kiš] / 4. [34 (x) ] sahar-ra / 5. [tùn.ka]ra bí-sì /} 6. bàd-bi / 7. ì.gul.gul. / 8. zà-a-ab-ba-ka-še / 9. má-me-luh-ha.ki / 10. mámá-gan.ki / 11. má-dilmun.ki

1 sár-ru-gi / 2. lugal / 3. kišs / 4. 34 rec 169 / 5. iš gul. / 8. a-dì-ma / 9. pu-ti / 10. ti-a-am-ti/11. má me-luh-ha/12. má má-gan. ki/ 13 má dilmun.ki

"Sargon, rey de Kiš, ganador de 34 batallas, destructor de las murallas hasta la orilla del mar. Hizo amarrar en los muelles de Acad los barcos de Meluhha, los barcos de Magan y los barcos de Dilmun"13.

Así, con Sargón inauguramos la famosa asociación "Dilmun, Magan y Meluhha" que se extenderá a lo largo del III milenio a.C. y que será rescatada por los reyes neoasirios del I milenio a.C. ${ }^{14}$. En realidad, son los barcos -má- con los materiales provenientes de esta ruta

\footnotetext{
${ }^{9}$ S. MÉRY, "Origine et production des récipients de terre cuite dans la péninsule d'Oman à l'Âge du Bronze" Paléorient 17, 1991, pp. 51-78; S. MÉRY, "La céramique Mésopotamienne de Période Hafit" en S. Méry (ed.), Les céramiques d'Oman et l'Asie moyenne. Une archéologie des échanges à l'Âge du Bronze. Monographies $d u$ CNRS 23, 2000, pp. 170-189.

${ }^{10}$ E. SOLLBERGER, Inscriptions Royales Sumeriennes et Akkadiennes, 1971, Cap. I y II, pp. 44-45.

${ }^{11}$ E. SOLLBERGER, 1971, p. 94.

${ }_{12}$ Textos Ni 3200, (textos de Nippur y Estambul) y CBS 13972, (Textos babilónicos de Pensilvania). Vid. D. FRAYNE, The royal Inscriptions of Mesopotamia early periods volumen 2. Sargonic and Gutian periods (2334-2113 BC), 1993, pp. 27-29.

${ }^{13}$ E. SOLLBERGER, 1971, p. 97.

${ }^{14}$ C. del CERRO, "Gran rey, rey del mundo, rey de Asiria...¿¿Rey de reyes de las tierras de Dilmun, Magan y Meluhhą?" Isimu 16, 2013, p. 54.
} 
comercial $^{15}$, los protagonistas de esta afirmación de Sargón. En cierta forma, garantizar la llegada de esos materiales justificaría la toma de aquellos territorios sureños por los que los bienes llegarían a Acad.

Maništušu (2269-2255 a.C.) nos ha dejado sobre una tablilla y varios fragmentos en piedra, el recuerdo de una campaña exitosa en Irán. El texto dice que el rey acadio construyó barcos para cruzar después el Mar Inferior, donde una fuerza de treinta y dos ciudades se reunió para la batalla. Maništušu resulta victorioso dominando ciudades y reyes. Tras unas líneas complicadas de entender, el relato indica que el rey persiguió a las tropas enemigas hasta la región de minas de metal y se llevó piedra negra de las montañas, cargada en sus barcos hasta Acad ${ }^{16}$. Una vez allí, utiliza esa piedra para realizar una estatua de sí mismo y dedicarla a Enlil. La inscripción es estándar y tenemos copias de la misma en los museos Nacional de Iraq, Filadelfia, Estambul, Louvre y Británico, líneas 1- $63^{17}$

1.ma-an-ís-tu-su / 2. lugal / 3. kišs / 4. ì-nu / 5. an-ša-an. ki / 6. ù / 7. ši ${ }_{4}-r i ́-h u-$ um.ki / 8. sag.giš.ra-ni / 9. ti-a-am-tàm / 10. sa-pil-tàm / 11. má.má giš.la-e / 12. u-sa-bi-ir / 13. uru.ki.uru.ki / 14. a-bar-ti / 15. ti-a-am-tim / 16.32 a-na / 17. $\operatorname{rec} 169$ / 18. ip-hu-ru-nim-ma / 19. išs ${ }_{11}$-ar / 20. ù / 21. uru.ki.uru.ki- su-nu / 22. sag.giš.ra / 23. en.en-su-un / 24. [u-s]a-am-[q]í-it /25. ù / 26. is-tu[m-ma]/ 27. i[d-ké-ás-su-nu-ni-ma] / 28. a-dì-ma / 30. hu-rí kù / 31. il-qù-tu / 32. a-barti / 33. ti-a-am-tim / 34. sa-pil-tim/35. $\mathbf{n a}_{4} \cdot \mathbf{n a}_{4}$-su-nu-gi ${ }_{6} / 36$. - i-pu-lam-ma / 37. in má.má /38. i-ŝa-[na-ma] / 39. in ka-rí-<im >/40. ši a-kà-dè.ki / 41. ir-ku $-u s$ / 42. dùl-su / 43. ib-ni / 44. a-na / 45. [ ${ }^{\mathrm{d} e n-l i ́ l] ~ / ~ 46 . ~ a . m u . r u ~ / ~ 47 . ~}{ }^{\mathrm{d} u t u ~ / ~ 48 . ~ u ̀ ~ / 49 . ~}$ il-a-ba $/$ / 50. ú-má / 51. la sú-ra-tum / 52. lu kí-ni-is-ma / 53. ša dub / 54. su $-a$ / 55. u-sá-sà-ku-ni / 56. ${ }^{[\mathrm{d}]}$ en-líl / 57. ù / 58. ${ }^{\mathrm{d}} \mathbf{u t u} /$ 59. suĥǔs- sú / 60. li-sú-ḩa / 61. ̀̀ / 62. še.numun-sú / 63. li-il-qù-tá

1.“Maništušu, rey de Kiš, 4. cuando conquistó Anšan y Šerihum, 9. atravesó el Mar Inferior en barcos... 13. Las ciudades del otro lado del mar, treinta y dos se aliaron para la batalla, pero él triunfó 20 . y conquistó sus ciudades y a todos sus príncipes eliminó y 25. [...] hasta las minas de plata. 31. Él extrajo las piedras negras de las montañas más allá del Mar Inferior y las cargó en los barcos y les hice amarrar en los muelles de Acad. 42. Hizo diseñar una estatua (de él) y la dediqué al dios Enlil. Por Šamaš y Aba, juro que no miento, es absolutamente cierto 51. El que borre esta inscripción, que Enlil y Šamaš le arranquen sus cimientos y destruyan su progenie"18.

Este texto supone la primera mención de una expedición marítima mesopotámica, el relato tiene todos los ingredientes para que podamos situar esta empresa frente a las costas de la Península de Omán 19: "el otro lado del mar", "más allá del Mar Inferior" "las minas de plata" y "las piedras negras de las montañas".

\footnotetext{
${ }^{15}$ G. PETTINATO, "El commercio con l'estero della Mesopotamia meridionale nel 3 millenio av. Cr, alla luce delle fonti letterarie e lessicali sumeriche" Mesopotamia 7, 1972, passim.

${ }^{16}$ De los metales que se extrajeron en Magan y de los que existen, pero no se explotaron, así como de sus piedras, dejaremos constancia en el siguiente apartado de nuestro trabajo.

${ }^{17}$ D. FRAYNE, 1993, pp. 74-77.

${ }^{18}$ E. SOLLBERGER, 1971, p. 104.

${ }^{19}$ D.T. POTTS, Mesopotamian and the east. An Archaeological and Historical Study of Foreign relations 34002000 BC, 1994, p. 105.
} 
Pero la primera vez que aparece el término Magan aislado, má-gan.ki, (Maništušu nos había dejado con la duda) es de la mano de Naram Sin (2254-2218 a.C.), en el momento en el que el monarca acadio nos informa de haber organizado un ataque contra Magan, fechado aproximadamente en el 2200 a.C ${ }^{20}$. Uno de los relatos que podemos leer se encuentra en el anverso de la tablilla HS 1954+1955+2499+2506 que forma parte de la Hilprecht Sammlung der Schiller Universität von Jena ${ }^{21}$, donde entre líneas muy mal conservadas leemos:

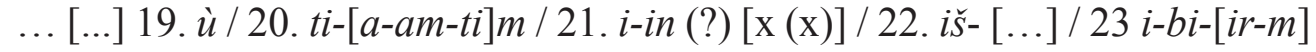
$a / 24 . m[a ́$-gan.k]i / 25. qáb-li / 26. ti-[a]-am-ti / 27. sag.giš.ra / 28. ù / 29. gišs. tukul- ki-su / 30. $i[n]$ ti-a-am-tim / 31. sa-píl-tim / 32. ì.luh “ ... [...] 19-27 además él cruzó...el Mar (Inferior) y conquistó M[agan], en el medio del mar / 28- 32 y lavó sus armas en el Mar inferior"

Tras el acontecimiento que conocemos como La gran revuelta, Naram Sin incorpora a la titulatura real la fórmula "el victorioso en nueve batallas en un año" refiriéndose, suponemos, a todas sus vitorias tras el alzamiento ${ }^{22}$. El texto lo encontramos además escrito una inscripción en Marad ${ }^{23}$, en la base de una estatua en Bassetki ${ }^{24}$, cerca de Mosul, tablillas en Nippur ${ }^{25}$, y en la base de una estatua hallada en la acrópolis de Susa $^{26}$ que es la más completa, una de las primeras en las que el rey aparece con determinativo de divinidad y la que nos lleva a Magan ${ }^{27}$ :

Col I. 1. ${ }^{d}$ na-ra-am-den.zu / 2. da-núm / 3. lugal / 4. ki-ib-ra-tim / 5. ar-ba-im / 6. ša-ir / 7. 10 lal 1 rec 169 / 8. [i]n mu 1 / 9. [i] s-tum / 10. [re]c 169 rec 169 / $11\left[s u_{4}\right]$-un-ti / 12. [iš-a]r-ru / 13. ù / 14. [sar-ri] $]-s u ́-[n u] 3 / 15 .[i-i k-m i-m a] /$ 16. $\left[\right.$ mah-rí-is] / 17. [ ${ }^{\mathrm{d} e n-l i ́ l] / 18 . ~[u-s a-r i ́-i b] / 19 . . . . . . . . . . ~}$

Col II. 1. má-gan.ki / 2. sag.giš.ra / 3. ù / 4. ma-ni-u[m] / 5. e[n] / 6. má-gan. [ki] / 7. su.du ${ }_{8} \cdot[\mathbf{a}] /$ 8. in sa.dú-su-nu / 9. na $\mathbf{4}_{\mathbf{4}} \cdot \mathbf{n a}_{4} \cdot \mathbf{e}-\mathbf{s i} \mathbf{1}_{11}-\mathbf{i}[\mathrm{m}] /$ 10. i-pu-lam-ma / 11. a-na / 12. a-kà-dè-ki / 13. uru.ki-su / 14. u-bí-lam-ma / 15. dúl.su / 16. ib-ni

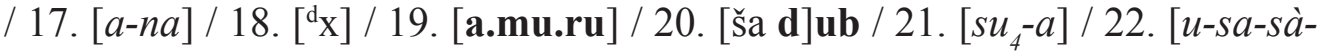
$k u]-n i / 23$. [ $\left.{ }^{\mathrm{d} u t u}\right] / 24 . \grave{u} / 25$. [dingir (?) a-kà]-d[è]-ki / 26. [suh]uš-su / 27. [l] i-sú-h̆a / 28. ù / 29. še.numun-su / 30. 1i-il-qù-tá

Col I 1-8 "Naram Sin, rey fuerte, rey de las cuatro regiones, victorioso en nueve batallas [e]n un año / 9-12 [Tr]as haber [ga]nado en [e]sas [ba]tallas/ 13-18. [capturó] sus tres [reyes] [y los trajo ante Enlil]

\footnotetext{
${ }^{20}$ J.J. GLASSNER, "Mesopotamian textual evidence on Magan/Makan in the late 3rd Millennium BC" en P. Costa y M.Tosi (ed.), Oman studies. Papers on the archaeology and history of Oman, Serie Orientale Roma 63, 1989, p. 181.

${ }^{21}$ La unión de las tablillas halladas en Nippur HS 1954+1955+2499+2506 y depositadas en Jena, ha permitido a B. Foster publicar una edición crítica del texto dentro del Royal Inscriptions of Mesopotamia Project. B. FOSTER, "Naram-Sin in Martu and Magan" ARRIM 8, 1990, passim.

${ }^{22}$ D. FRAYNE, 1993, p.111.

${ }^{23}$ I.J. GLEB y B. KIENAST, Die Altakkdischen Königsinschriften der Dritten Jahrtausends Vor. Chr., FAOS 7 , 1990, pp. 102-103.

${ }^{24}$ ABDUL-HADI AL-FOUADI, "Bassetki Statue with Old Akkadian Inscription of Naram-Sin of Agade (B.C. 2291-2255)" Sumer 32, 1976, pp. 63-75.

${ }^{25}$ Vid. nota 21.

${ }^{26}$ Número de catálogo del Louvre Sb 52, depositada en el Département des Antiquités orientales por J. de Morgan, vid. http://carteles.louvre.fr/carteles/visite?srv=car_not_frame\&idNotice=11959\&langue=

${ }^{27}$ D. FRAYNE, 1993, pp. 116-118.
} 
Col II 1-4. Él subyugó Magan y capturó a Maniu[m], re[y]/en de Magan/ 8-14. de sus montañas él extrajo bloques de diorita y los trajo a Acad, su ciudad, e / 15-16. hizo una estatua de sí mismo / 17-19. [la dedicó al dios X] 20-22 [el que quit]e [esta in]scripción / 23-30. Que el dios Šamaš y el dios de Acad arranque sus cimientos y destruya su progenie" 28

El texto esta vez sí es claro: 'ma-ni-um en má-gan.ki ${ }^{29}$. Y esta es la primera vez que un rey, lo que implica la existencia de un reino, gobierna en Magan, ya en el S. XXII a.C. No tendremos muchas ocasiones de repetir esta idea en los textos mesopotámicos, salvo en épocas Isin Larsa y neoasiria.

Aunque ni los textos acadios, ni los neosumerios, ni la arqueología en la Península de Omán indiquen que la presencia acadia sacudiera la vida diaria de los habitantes de Magan, la repercusión de la empresa de Naram Sin perduró, porque textos posteriores en el tiempo nos recuerdan la campaña del rey acadio mencionando al rey de Magan. En el texto paleobabilónico, probablemente de carácter escolar ${ }^{30}$ conocido como la Insurrección general contra Naram Sin, entre una lista de rebeldes, nos encontramos con 'ma-un-um lugal má.gan.na.ki "Maunum, rey de Magan" 31 .

Por último, la crónica neobabilónica que conocemos como Crónica de los primeros reyes $^{32}$ recuerda que Naram Sin llegó a Magan y capturó a Mannudannu de Magan:

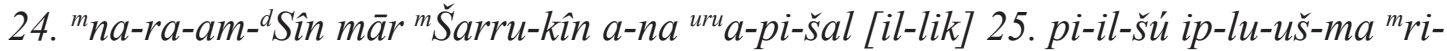

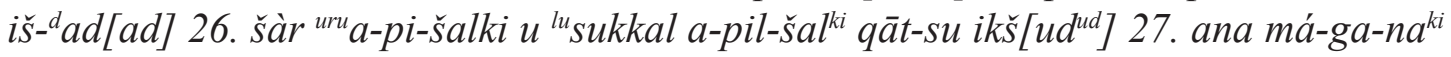
il-lik-ma mam-nu-da-an-nu šàr má-gan ${ }^{k i}$ [qāt-su ikšudud]

24. Naram Sin, hijo de Sargon [marchó] a Apišal 25-26. Hizo una brecha (en la muralla) y capturó a Reš-Ad[ad] rey de Apišal, y el visir de Apišal 27. Él marchó contra Magan y [capturó] a Mannudannu, rey de Magan

El botín debió de ser fascinante si atendemos a la cantidad de vasos de alabastro, calcita o dolerita hallados en Susa, Ur y otros lugares no identificados de $\operatorname{Iraq}^{33}$ con inscripciones de Naram Sin. Los cuencos (i.e NBC 2527, Universidad de Yale, BLMJ 929, Museo de la Biblia de Jerusalén o CBS 14951+14952, Universidad de Filadelfia) tienen la misma inscripción ${ }^{34}$ :

\section{1. na-ra-am-den.zu / 2. lugal / 3. ki-ib-ra-tim / 4. ar-ba-im / 5. bur / 6. nam-ra- ak / 7. má-gan.ki \\ 1-4 "Naram Sin, rey de las cuatro regiones 5-7 vaso del botín de Magan".}

Aparte de las inscripciones reales, que son los documentos más conocidos, escasos textos de naturaleza administrativa y comercial en época acadia permiten a B.R. Foster ${ }^{35}$

\footnotetext{
${ }^{28}$ Vease también D.T. POTTS, The Arabian Gulf in Antiquity vol I, 1994b, pp. 136-137.

${ }^{29}$ Vid Narāmsu'en-Inschr. a 2:SAK 166:h recogida en D.O. EDZARD, G. FARBER y E. SOLLBERGER, Répertoire Géographique des Textes Cunéiformes I. TAVO, Reihe 13, 7/1, 1977, pp. 113-114.

${ }^{30}$ J.G. WESTENHOLZ, Legends of the Kings of Akkade. The Texts, 1997, p. 239.

${ }^{31}$ La tablilla ya había sido estudiada por A. Boissier, quien lee en la línea 30 del reverso "Manum king of Makkan" A. BOISSIER, "Inscription de Narâm-Sin” RA 16, 1919, pp. 157-164. El texto completo y su traducción al inglés puede leerse también en J.G. WESTENHOLZ, 1997, pp. 239-245.

${ }^{32}$ A.K. GRAYSON, Assyrian and Babylonian Chronicles, 2000, p. 154.

${ }^{33}$ D.T. POTTS, 1994b, p. 113.

${ }^{34}$ D. FRAYNE, 1993, pp. 99-100.

${ }^{35}$ B. R. FOSTER, "Commercial activity in sargonic Mesopotamia” Iraq 39, 1977, pp. 38-39.
} 
realizar un mapa con modelos comerciales en época acadia muy ligado a la zona geografía dónde se localiza cada ciudad mesopotámica, es decir, resaltando la regionalización interna de Mesopotamia que ya se distinguía a lo largo del III milenio a.C. ${ }^{36}$. Así, en época acadia Lagaš se vincula al pescado y la sal o Umma se especializa en aceites aromáticos. Es precisamente el yacimiento de Umma el que nos permite ver mejor los contactos de Acad con el exterior, en el caso de Magan solo dos ejemplos nos ilustran: la presencia un mercader de Magan que exporta cerveza de $\mathrm{Umma}^{37}$ y la mención expresa del cobre de Magan $^{38}$ en dos textos de Tello.

\section{Las Montañas de Omán}

Los textos acadios no solo nos hablan de Magan y de su reino, sino que insisten en la elaboración de estatuas erigidas con las "piedras negras" traídas desde Magan. Durante el periodo acadio las rocas ígneas, oscuras, son las que se utilizan en mayor medida para la escultura monumental. De los veintiséis monumentos acadios (estelas, estatuas y basas) hallados por los franceses en Susa, diecisiete (73\%) fueron tallados en una roca negra, olivinagabbro $^{39}$, que habitualmente conocemos como diorita ${ }^{\text {na4 }}{ }^{2} \mathbf{e s i} / u s ̌ \hat{u}$. Esta piedra es habitual en las montañas de Omán y no podemos olvidar la frase de Naram Sin "Él subyugó Magan y capturó a Manitan, en de Magan, él trajo bloques de diorita de sus montañas".

Pero ¿qué son en realidad esas montañas negras que señalaban a Maništušu el rumbo y le ofrecían sus productos a Naram Sin? ¿realmente podría haber visto una expedición acadia esta cordillera? No sería extraño si tenemos en cuenta que a lo largo de toda la costa de la Península de Omán las montañas se alzan majestuosamente, peladas de vegetación, y mostrando su llamativo color oscuro desde cada punto del Golfo Pérsico y el Mar de Omán. No es posible obviarlas ni dejar de señalar su monumental mole desde un barco que se acerque al estrecho de Ormuz.

El color de las montañas de Omán se debe no tanto a afloramientos de dolerita (gabro o diorita), cosa que podríamos pensar a tenor de los textos acadios, sino a las grandes extensiones de peridotitas que quedan a la vista, una roca ígnea que forma el manto terrestre, pero que en Omán aflora a la superficie durante cientos de kilómetros.

La cadena de al Hayyar recorre la Península de Omán de norte a sur, es una verdadera columna vertebral donde asentamientos, materias primas y riqueza de vegetación se dan la mano. Forma un arco de $700 \mathrm{~km}$ de largo y $150 \mathrm{~km}$ de ancho paralelo al golfo de Omán. Se extiende desde la península de Musandam y el estrecho de Ormuz, al norte, hasta Ras al Hadd, en el sureste, con alturas que oscilan normalmente entre 500 y $1.500 \mathrm{~m}$ destacando la altura del yebel Ajdar, cerca de Nizwa, con $3.000 \mathrm{~m}^{40}$.

Desde el punto de vista geológico las Montañas de Omán presentan seis grupos de secuencias rocosas ${ }^{41}$, pero anotamos aquí solo los dos grandes complejos que nos interesan en esta exposición:

\footnotetext{
${ }^{36}$ H. E. W. CRAWFORD, "Mesopotamia's Invisible Exports in the Third Millennium B.C.” World Archaeology 5/2, 1973, p. 233.

${ }^{37}$ Vid. Serie Manchester Cuneiform Studies (MCS) 9, 245.

${ }^{38}$ Vid. Inventaire des tablettes de Tello conservées au Musée Impérial Ottoman (ITT) I, 1422 y II, 2846, Publicadas por primera vez por F. THUREAU-DAGIN ET AL, en París, 1910 y 1912.

${ }^{39}$ D.T. POTTS, 1994b, p. 183.

${ }^{40}$ L.R. WEEKS, Pre-Islamic Metallurgy of the Gulf, Technology, Trade and the Bronze Age World, 2003, p. 10.

${ }^{41}$ M.C. ZIOLKOWSKI, "The Soft Stone Vessels from Sharm, Fujeirah, United Arab Emirates" AAE 12, 2001, pp. 25-30.
} 
1. Complejo de Hawasina donde abunda el mineral de cobre, con presencia de gabros y peridotitas ${ }^{42}$, éstas surgen desde Ibra to Khor Fakkan, en la costa, desde donde los bloques podrían ser trasladados ${ }^{43}$. El depósito que contiene además altos niveles de oro y plata, sin embargo y hasta ahora, no hay evidencia arqueológica de la explotación minera de ambos metales esta zona en la Antigüedad ${ }^{44}$. Actualmente el oro es extraído en Raka (Omán) electrónicamente del mineral de cobre, pero solo es posible realizar esta extracción con tecnología moderna ${ }^{45}$.

2. Complejo ofiolítico Sumā'il, roto en doce bloques intactos con formas variadas como resultado de la erosión, pero que tiene en total $600 \mathrm{~km}$ de largo por $150 \mathrm{~km}$ de ancho. Cubre en total un área de $20.000 \mathrm{Km}^{2}$ de Dibba a Sūr, es por tanto la unidad geológica más grande de la cadena de al Hayyar. Se admite que Sumā’il es la mayor capa ofiolítica expuesta del mundo ${ }^{46}$. En él se hallan las piedras blandas (esteatita, clorita o serpentina) que se demandan incluso desde Mesopotamia, así como la mayor parte de los depósitos de cobre del sureste de Arabia.

Pero los textos acadios, y más adelante los neosumerios, insisten aún más en otro producto proveniente de Magan, un bien tan habitual en las Montañas de Omán que la región queda registrada en ocasiones como el país del cobre, urudu/erû, y aunque los objetos elaborados en cobre son escasos en Mesopotamia lo cierto es que dos de ellos son excepcionales y nos permiten conocer la pericia de los artesanos acadios: la cabeza de Nínive (conocida comúnmente como cabeza de Sargón) ${ }^{47}$ y la estatua de Bassetki ${ }^{48}$.

La primera investigación sobre la producción de cobre en Omán comenzó en un lejano 1928 cuando H. Peake ${ }^{49}$ indicó que éste era el cobre de la Magan de los textos mesopotámicos. Unos sesenta años después, en los años setenta del S. XX, comenzaron a las grandes campañas de prospecciones geomineras y arqueológicas llevadas a cabo en la Península. La primera fue conducida por la Universidad de Harvard y el Instituto de Paleontología de Roma ${ }^{50}$.

A principios de los años ochenta del siglo pasado los franceses del CNRS se sumaron a este estudio. Con sus análisis concluyeron que el comercio del cobre de Magan significó una verdadera interacción entre las comunidades del III milenio a.C. de la Península con sus contemporáneas de Mesopotamia e Irán, y supuso un papel importante en el desarrollo de las sociedades de la Península de Omán ${ }^{51}$. Además, desde enero de 1977 el Museo de minero de Bochum $^{52}$ en colaboración con el Departamento de Antigüedades de la Universidad de

\footnotetext{
${ }^{42}$ FAISAL BIN ALI AL SAID, “The Environmental Setting of the Sohar Region” JOS 9, 1987, pp. 30-33.

${ }^{43}$ P. YULE y I. GUBA, "Did the Ancient Mesopotamian Royal Stone Originate in Oman?" Adumatu, 4, 2001, p. 44.

${ }^{44}$ L.R. WEEKS, 2003, p. 14.

${ }^{45}$ P. YULE y I. GUBA, p. 42.

${ }^{46}$ K.M. GOODENOUGH ET AL., "Records of Ocean Growth and Destruction in the Oman-UAE Ophiolite" Elements 10, 2014, p. 109.

${ }^{47}$ C. NYLANDER, "Earless in Nineveh: Who Mutilated "Sargon's" Head?” AJA 84/3, 1980, passim.

${ }^{48}$ P.R.S. MOOREY, "The archaeological evidence for metallurgy and related technologies in Mesopotamia" Iraq 44, 1982, pp. 35-36.

${ }^{49}$ H. PEAKE, "The Copper Mountain of Magan" Antiquity 2, 1928, passim.

${ }^{50}$ J.H. HUMPHRIES, "Harvard Archaeological survey in Oman: II- Some later prehistoric sites in the Sultanate de Oman" PSAS 4, 1974, passim.

${ }^{51}$ L.R. WEEKS, 2003, pp. 19-20, cap. 2.4.4 Analyses by The Centre National de la Recherche Scientifique (CNRS), France.

${ }^{52}$ El resultado más inmediato y completo fue la obra de A. HAUPTMANN, 5000 Jahre Kupfer in Oman; Band 1: Die Entwicklung der Kupfermetallurgie vom 3. Jahrtausend bis zur Neuzeit Der Anschnitt, Beiheft 4, editada en 1985 en Bochum.
} 
Nápoles comenzó una de las investigaciones más extensas realizadas sobre las montañas de Omán ${ }^{53}$. Más de ciento cincuenta minas y lugares de fundición fueron documentados y datados desde el III milenio a.C. hasta época islámica. En total estimaron que existen cincuenta grandes depósitos de cobre en las montañas y más de cien de menor importancia. La mayor parte parecen haber sido explotados en época islámica, pero sin duda algunos ya lo fueron desde el III milenio a.C., puesto que las tumbas Umm an-Nar (cultura omaní contemporánea a la acadia) lo corroboran, así como el asentamiento de Maysar ${ }^{54}$ o los muchos lugares de extracción diseminados por las montañas. Todas estas prospecciones por las montañas de Omán nos han demostrado que ciertas áreas como Lasail ${ }^{55}$, 'Arja ${ }^{56} \mathrm{o} \mathrm{Masjad}^{57}$ -en la región de Sohar ${ }^{58}$ - han estado ligadas al menos desde el III milenio a.C. a la explotación minera, y que éstas son las tres áreas donde se hallan los depósitos de mineral de cobre más grandes de la Península. Sin embargo, los estudios más recientes vienen de la otra vertiente de las montañas de Omán, del emirato de Sharjah, donde queda localizado el Wādi al-Hilo y el yacimiento denominado HLO $1^{59}$ cuya datación por radiocarbono fechó las estructuras entre 2336-2203 a.C.; época acadia en Mesopotamia, mientras que la cerámica Umm an Nar corroboraba la datación ${ }^{60}$. La excavación del lugar de fundición y trabajo permitió encontrar el único lingote ${ }^{61}$ de cobre $-4,6 \mathrm{~kg}$ - encontrado hasta ahora en esta vertiente de las montañas ${ }^{62}$ y el único in situ, en el sureste de Arabia, y una cantidad de escorias tal que sus excavadores calculan al menos la producción en un mínimo de 28 toneladas de cobre en el área ${ }^{63}$.

\section{Asentamientos y cultura material en la Península de Omán. La Cultura Umm an Nar}

Cuando los textos acadios ya nos hablan de un reino y de un rey, ma-ni-um, en Magan, entonces tropezamos con la realidad arqueológica de la Península de Omán. Desde aquí

${ }^{53}$ G. WEISGERBER, "Evidence of Ancient Mining Sites in Oman: a Preliminary Report” JOS 4, 1978, pp. 15-28, Taf. 11a-26.

54 A. HAUPTMANN y G. WEISGERBER, "Third millenium BC copper production in Oman" Revue d'Archéométrie 1, 1981, pp. 131-133.

${ }^{55}$ G. WEISGERBER, 1978, pp. 21-22.

${ }^{56}$ T.J. COSTA y P.M. WILKINSON, "Settlement and Copper Exploitation in the 'Arja area" JOS 9, passim; G. WEISGERBER, “Archaeological Evidence of Copper Exploitation at 'Arja” JOS 9, 1987a, passim.

${ }^{57}$ M. TOSI, "Notes on the Distribution and Exploitation of Natural Resources in Ancient Oman" JOS 1, 1976, pp. 187-206.

${ }^{58}$ T.J. COSTA y P.M. WILKINSON, “The Environmental Setting of the Sohar Region” JOS 9, 1987b, passim.

${ }^{59}$ J. KUTTERER y S.A. JASIM, "First report on the copper-smelting site HLO-1 in Wādī al-Hilo, UAE" PSAS 39, 2009, passim.

${ }^{60}$ J. KUTTERER y S.A. JASIM, 2009, pp. 249-250.

${ }^{61}$ Este lingote fue dividido en dos partes por la Autoridad Arqueológica de Sharjah para estudiar su interior que consiste en cobre puro en un 99.5\% con trazas de hierro, níquel y arsénico (J. KUTTERER y S.A. JASIM, 2009, p. 250). Una de las partes, con $3 \mathrm{~kg}$ de peso, fue trasladada a Madrid para formar parte de los objetos que se expusieron en el Museo Arqueológico Nacional con motivo la exposición En los confines del Oriente Próximo El hallazgo moderno del país de Magán. Veinte años de descubrimientos del Departamento de Antigüedades de Sharjah, la comunidad cientifica internacional y la misión de la Universidad Autónoma de Madrid en Sharjah (Emiratos Árabes Unidos), cuyo catálogo fue editado por J.Ma . CÓRDOBA (Coord.) en 2016, quedando la relación de piezas a cargo de C. del Cerro, vid. p. 42 y fig. 12, p. 51.

${ }^{62}$ Lingotes de cobre de este tipo, con forma planoconvexa, ya habían sido hallados en Omán en 1983, en al Aqir cerca de Bahla pero estudiados más tarde y fuera de contexto por G. Weisgerber, y con una datación un poco más tardía, alrededor de 2000 a.C. (G. WEISGERBER y P. YULE, “Al-Aqir near Bahlā’ an Early Bronze Age dam site with planoconvex 'copper' ingots" AAE 14, 2003, p. 51; F. BEGEMANN ET AL., "Lead isotope and chemical signature of copper from Oman and its occurrence in Mesopotamia and sites on the Arabian Gulf coast" $A A E 21,2010$, p. 138). Varios fragmentos de lingotes y un solo lingote completo se encontraron a su vez en Maysar, en la llamada House 4 con la misma cronología (G. WEISGERBER y P. YULE, 2003, p. 48.) ${ }^{63}$ J. KUTTERER y S.A. JASIM, 2009, p. 251. 
no hay textos que autoricen devolver la mirada a Acad, lo que puede permitir a algunos historiadores apostar por la teoría de W. Heimpel ${ }^{64}$ y seguir dudando de la ubicación de Magan en época acadia ${ }^{65}$. Pero la Península muestra una riqueza arqueológica que impone a todo aquel que simplemente se asome a ella, porque se asombrará al descubrir la cultura contemporánea a la acadia en el otro extremo del Golfo; la cultura Umm an-Nar (Fig. 2). La cultura Umm an Nar ha sido estudiada fundamentalmente desde el punto de vista funerario ${ }^{66}$, sus tumbas colectivas aparecen en toda la región pero algunos yacimientos nos permiten saber que poseyó una población sedentarizada en el III milenio a.C. al menos en la isla de Umm an $\mathrm{Nar}^{67}$, en los yacimientos costeros de tell Abraq ${ }^{68}$ y Ras al Hadd (HD-6) ${ }^{69}$ y en los oasis del interior de la península (Hili, al Ain $)^{70}$. El motivo de la incipiente sedentarización en época Umm an Nar provendría de la consolidación de la agricultura de oasis a pequeña escala ${ }^{71}$, ya documentada mitad del III milenio a.C. en yacimientos como Hili 8. Y esa agricultura sería posible, en el caso de los oasis del interior, por la existencia de una capa freática muy cercana a la superficie captada a través de $\operatorname{pozos}^{72}$, que permitiría el cultivo de los oasis y la explotación, nunca intensiva, de sus alrededores ${ }^{73}$. La agricultura de los oasis siempre se basó en el cultivo de la palmera datilera (Phoenix dactylifera ${ }^{74}$ ) bajo cuya sombra crecían cereales verduras o legumbres ${ }^{75}$.

\footnotetext{
${ }^{64}$ W. HEIMPEL, "A first step in the diorite question” $R A 76,1982$, p. 66.

${ }^{65}$ J.J Glassner, 1989, pp. 184-186; B. Foster, The Age of Agade Inventing Empire in ancient Mesopotamia, 2016, p. 100.

${ }^{66}$ Comenzando por los primeros estudios de los daneses (K. FRIFELT, The island of Umm an-Nar, vol I: third millennium graves. Jutland Archaeological Society Publications 26 /1, 1991 y K. FRIFELT, The island of Umm an-Nar, vol II: the third millennium settlement. Jutland Archaeological Society Publications, 26/2, 1995) a los últimos resultados publicados, pertenecientes a las necrópolis de Mleiha-al Madam en el emirato de Sharjah (S.A. JASIM y E. YOUSIF, "Bronze Age Tombs from Jebel Faya, Emirate of Sharjah” Sharjah Archaeology 15, 2016, pp. 8-17; S.A. JASIM, M. UERPMANN y H.P. UERPMANN, H. Mleiha: the unwritten History. Sharjah, 2016, pp. 41-59.)

${ }^{67}$ W.Y. AL TIKRITI, Archaeology of Umm an-Nar island: 1959-2009, 2011, Abu Dhabi. Umm an-Nar es una pequeña isla cerca de la ciudad de Abu Dhabi. Desde 1985 una misión danesa trabajó en la zona excavando un asentamiento formado por casas de planta cuadrada con fundaciones de piedra. Además, el equipo encontró una necrópolis con cuarenta y nueve tumbas de las que siete fueron excavadas por el equipo danés, datadas a caballo entre del Dinástico Temprano y la época acadia.

${ }^{68}$ D.T. POTTS, Ancient Magan. The secrets of Tell Abraq, 2000. El tell se halla en la linea divisoria que separa Sharjah de Umm al Qaiwain y fue excavado primero por D.T Potts, que recogió las primeras campañas en D.T. POTTS, "Four seasons of excavation at Tell Abraq (1989-1993)" PSAS 23, 1993b, pp. 117-126, y posteriormente y hasta la actualidad por P. Magee, P. MAGEE, ET AL, "Report on Excavations at Tell Abraq, Emirate of Sharjah, United Arab Emirates 2010-2013" Sharjah Antiquities 14, 2015, pp. 5-29. El tell tiene igualmente niveles de época acadia.

${ }^{69}$ P. MAGEE, The Archaeology of Arabian Peninsula. Adaptation and Social Formation from Neolithic to the Iron Age, 2014, pp. 96-87.

${ }^{70}$ S. CLEUZIOU, Archeologie aux Emirats Arabs Unis. Vol I-III. Al-Ain, 1977-1979; S. CLEUZIOU, S. MÉRY y B. VOGT, Protohistorie de l'oasis d'Al-Ain, Travaux de la Mission archéologique française à Abou dhabi (Emirats Arabes Unis). BAR International Series 2227, p. 2011.

${ }^{71} \mathrm{~S}$. CLEUZIOU y M. TOSI, In the shadow of ancestors. The prehistoric foundations of the early Arabian civilization in Oman, 2007, pp. 90-91.

${ }^{72}$ P. MAGEE, 2014, p. 95.

${ }^{73}$ S. CLEUZIOU, "Enjeux et Perspectives de la Recherche Archéologique dans la Péninsule Arabique, de la Fin de la Préhistoire à l'Âge du Bronze" Isimu 1, 1998, pp. 48-40.

${ }^{74}$ Huesos de dátil han sido documentados en el yacimiento de Dalma (Abu Dhabi) y fechados en el 5000 a.C., sin que podamos determinar aun si se trata de dátiles silvestres o cultivados; M. BEECH "Archaeobotanical evidence for Early Date consumption in the Arabian Gulf” en J.S. al Swaidi (dir.), The Date Palm. From traditional Resource to Green Wealth, 2003, p. 30.

${ }^{75}$ D.T. POTTS, "Date palms and date consumption in Eastern Arabia during the Bronze Age", en J. S. al Swaidi (dir.), The Date Palm. From traditional Resource to Green Wealth, 2003, p. 34. "Outside of the Omani
} 
Pero la excavación del sector 8 del yacimiento de Hili nos permite afinar más porque se trata de uno de los asentamientos mejor documentados de la Península de Omán en el III milenio a.C. ${ }^{76}$ En el yacimiento se hallaron dos áreas bien diferenciadas: la necrópolis y el asentamiento. Introducirnos ahora en esta necrópolis, de impresionantes y enormes tumbas circulares en piedra, sobre un plinto, con muros exteriores tallados y algunos con bajorelieves junto a la entrada de la tumba, nos separaría de nuestro objetivo, pero no podemos dejar de mencionar que en el interior de las tumbas se encontraron enterramientos colectivos con ajuar consistente en cerámica, armas de bronce y recipientes de clorita, cuya datación es 23002100 a.C.; época acadia en Mesopotamia ${ }^{77}$. La Tumba $\mathrm{N}$ de esta necrópolis además contenía una jarra típica del periodo acadio tardío en Mesopotamia, mientras que cerámica acadia es relativamente común en la costa, en la isla de Umm an $\mathrm{Nar}^{78}$. Se trata de vasijas destinadas a trasportar productos, quizás aceite, reutilizadas en ocasiones en los enterramientos ${ }^{79}$.

Muy cerca del área funeraria de Hili, una misión francesa dirigida por S. Cleuziou, situada ya en el yacimiento en 1977, excavó en Hili 8 una torre, alrededor de la cual se situaba el poblado. Tres edificios que parecen fortificaciones, refugios colectivos y residencia de los jefes locales, fueron desenterrados ${ }^{80}$. La cronología de Hili 8 muestra una ocupación desde época Yemdet Nașr a Ur III, siguiendo los siguientes niveles y sus correspondencias en términos mesopotámicos: Hili I = Yemdet Naṣr/Dinástico Temprano I, Hili II A-C1 = Dinástico Temprano II, Hili II C2 = Dinástico Temprano III, Hili II D-E = Acad y Hili IIF = Ur III. ${ }^{81}$ Pronto todo el material cerámico se puso en relación con paralelos en Irán y Baluchistán, en yacimientos como Bampur IV, tépé Yahya IVB, Sahr i-Sokhta IV, Ciudad I de Qalat al Bahrain, o el Templo I de Barbar, con una datación del 2700-2200 a.C. ${ }^{82}$

El asentamiento de Hili en el III milenio a.C., lo que incluye la época acadia, se asentó en los palmerales del actual al Ain, entre la zona de oasis, donde se explotaban las palmeras datileras, legumbres ${ }^{83}$, frutas como el melón ${ }^{84}$ y cereales como el trigo, la cebada, la avena, el mijo o el sorgo ${ }^{85}$. Hasta doce especies de plantas fueron halladas por el equipo francés en

highlands, the cultivation of much of the wheat, barley and alfalfa generally required a vegetational canopy, which was provide by date palms. Thus the date palms were not only important in they own right. Rather they provide the shade in which cereals and eventually fruits and vegetables could be grown by the early agricultural communities of eastern Arabia."

${ }^{76}$ C. del CERRO, "La península de Omán: sociedad y usos del entorno en los oasis de la antigua Magan”, Isimu 10, 2009, pp. 241-243.

${ }^{77}$ B. VOGT, "The Umm an Nar Tomb A at Hili North: a preliminary report on three seasons of excavation, 1982-1984" Archaeology of Unites Arab Emirates 4, 1985, p. 34.

${ }^{78}$ S. CLEUZIOU y S. MÉRY, "In between the great Powers in the Bronze Age Oman Peninsula" en S. CLEUZIOU ET AL. (eds.) Essays on the late Prehistory of the Arabian Peninsula, Serie Orientale Roma 93 , 2002, pp. 286-288.

${ }^{79}$ S. CLEUZIOU y S. MÉRY, 2002, p. 290.

${ }^{80}$ Tras las la octava campaña de excavaciones en Hili 8, S. Cleuziou mostró los resultados del yacimiento en el volumen V de la publicación Archaeology in the United Arab Emirates, donde presentaba el grueso de los hallazgos que habían tenido lugar entre la cuarta y la séptima campaña e incluía un informe preliminar de la octava. S. CLEUIZOU "Excavations at Hili 8; a preliminary report on the 4th and 7th campaigns" Archaeology in the United Arab Emirates V, 1989a, passim.

${ }^{81}$ S. CLEUZIOU, "The chronology of protohistoric Oman as seen from Hili”, en P. Costa y M. Tosi (eds.), Oman Studies. Serie Orientale Roma 63, 1989b, pp. 47-78.

${ }^{82}$ S. CLEUZIOU, 1998, passim.

${ }^{83}$ S. CLEUZIOU, 1998, pp. 48-49.

${ }^{84}$ D.T. POTTS, "Contributions to the agrarian history of Eastern Arabia II. The cultivars" AAE 5, 1994c, p. 238.

${ }^{85}$ S. CLEUZIOU, "Économie et société de la Penínsule d’Oman au IIIe millénaire; le rôle des analogies interculturelles" Colloques internationaux du CNRS 580, 1980, p. 349. 
semillas carbonizadas y e impresas en los adobes ${ }^{86}$. Los restos de fauna de ciento treinta y seis especies identificadas indican que la ganadería fue una actividad importante en Hili, ya que el $90 \%$ de la carne consumida era de animales domésticos y el $60 \%$ viene de la oveja o la cabra ${ }^{87}$.

Hili 8 nos muestra la primera utilización extensiva de la cerámica en el área. En la fase II E la mayor parte de las formas encontradas son jarras alargadas de labio biselado, jarras globulares y cuencos de labio grueso, pintados con líneas ondulantes ${ }^{88}$. Aparte del material cerámico, durante las campañas de excavación aparecieron vasos de serpentina y clorita, huesos y conchas, que adscribieron el nivel a la época acadia. ${ }^{89}$

El cobre es trabajado con seguridad a finales del III milenio a.C., ya que se ha encontrado un área de procesado del metal en la fase II E (que no es otra que la contemporánea a la época acadia), con moldes, crisoles y esquirlas de metal ${ }^{90}$, mientras que objetos como broches, cuchillos y cinceles aparecen en todos los niveles del asentamiento ${ }^{91}$. Una daga encontrada en esta fase nos indica que a mitad del milenio los objetos de cobre pueden tener una aleación con estaño, lo que implicaría un incipiente uso del bronce, que en ningún caso es habitual en este momento en Omán, donde el arsénico es más común y es más fácil que los objetos metálicos se fabriquen con un cobre arsenical. El poquito estaño que encontramos en los objetos de Hili podría llegar de Afganistán, y viajaría a través del Baluchistán y Sistán a las costas de la Península de Ománn² (Fig. 3).

La metalurgia del cobre también es conocida desde los yacimientos de Maysar 1, en las montañas, en la isla de Umm an Nar y en el asentamiento costero de Ras al Hadd. En Maysar $^{93}$ la metalurgia del cobre es una actividad continua desde el 2200 al 1900 a.C. Las minas se sitúan en el Wādi Samad mientras que a lo largo del valle se hallaron lugares con los hornos de fundición del mineral de cobre, junto a cinceles y varios fragmentos de lingotes ${ }^{94}$. Pero Maysar, a pesar de su innegable conexión con la metalurgia de cobre en la segunda mitad del III milenio a.C. significó una pequeña aportación respecto a todo el cobre que se explotó en este momento, porque las ciento cincuenta minas de cobre de las Montañas de Omán supusieron una explotación de 4.000 toneladas de cobre en época Umm an Nar. ${ }^{95}$

En la isla de Umm an Nar el cobre aparece en forma de anzuelos, cinceles, cuchillos, agujas y prendedores junto a las casas, además en algunas habitaciones parece que se trabaja el metal porque se hallaron moldes y crisoles ${ }^{96}$. En realidad, tal y como indica R.L. Weeks ${ }^{97}$ parece que el metal sufre un segundo procesado en la isla, aquí se le quitan las impurezas que quedaban y se da forma a los objetos, de ahí las pocas escorias halladas en el asentamiento. La isla de Ghanada, al norte de Umm an Nar, presenta un registro muy similar para este momento, incluyendo objetos mesopotámicos en el yacimiento ${ }^{98}$.

\footnotetext{
${ }^{86}$ D.T. POTTS, 1994 b, p. 80.

${ }^{87}$ M. UERPMANN y H.P. UERPMANN, "Animal economy during the Early Bronze Age in South-East Arabia" en E. Vila et al., Archaeozoology of the Near East 8, TMO, 49, Lyon, 2008, p. 468.

${ }^{88}$ S. CLEUZIOU, 1989a, pp. 76-77.

${ }^{89}$ S. CLEUZIOU, 1989a, tabla 1, p. 78.

${ }^{90}$ MUHAMMED ABDUL NAYEEM, The United Arab Emirates, 1994, p. 228.

${ }^{91}$ S. CLEUZIOU, 1989a, plate 33.

${ }^{92}$ S. CLEUZIOU, 1989a, p. 74.

${ }^{93}$ R.L. WEEKS, "Prehistoric Metallurgy at Tell Abraq, U.A.E” AAE 8, 1997, p. 17.

${ }^{94} \mathrm{Vid}$. nota 58.

${ }^{95}$ R.L. WEEKS, p. 17.

${ }^{96}$ K. FRIFELT, "On Prehistoric Settlement and Chronology of the Oman Peninsula" East and. West 25, 1975b, p. 365 .

${ }^{97}$ R.L. WEEKS, p. 18.

${ }^{98}$ R.L. WEEKS, p. 19.
} 
En la otra cosa de la Península, mirando al mar de Omán, se encuentra de Ras al Hadd ${ }^{99}$, sector 6; el yacimiento que mejor ilustra este periodo en el sultanato. El yacimiento consta de dos estructuras con varias habitaciones con restos de redes de pesca, anzuelos de cobre, prendedores, cuchillos y cinceles. La cerámica que acompañaba a los objetos de bronce era local e iraní ${ }^{100}$.

Entre los lugares de extracción de cobre en el interior y la costa, el traslado del metal hubo de hacerse, ya en época Unn an Nar, aprovechando un gran avance; la domesticación del asno (Equus asinus), hecho demostrado por los restos faunísticos de tell Abraq o Hili $8^{101}$.

\section{Conclusiones}

Los yacimientos de época Umm an Nar se cuentan a decenas, aunque aquí solo hayamos mencionados aquellos mejor publicados o los que nos permiten seguir mejor nuestro estudio y los que indican claramente niveles de época acadia. Se encuentran tanto en los lugares cercanos a las minas o donde se funde el cobre (Wādi al Ḥilo, Wādi Samad -en Maysar-, 'Arja, Masjad, Lasail) o en lugares con existencia de agua o posibilidades de cultivo en el piedemonte (Hili) o la costa (Umm an Nar, tell Abraq, Ghanada, Khor Fakkan o Ras al Hadd), lo cual les diferencia de los asentamientos islámicos siempre situados junto a la mina, en lo alto de la montaña ${ }^{102}$. Es evidente que la extracción de piedra y cobre formaba parte de las actividades de las comunidades Umm an Nar que, sin llegar a crear estados con un nivel de desarrollo equiparable al mesopotámico, podrían haber articulado la existencia de una autoridad que organizara esta producción para consumo interno y, a la luz de las fuentes mesopotámicas y de las cerámicas acadias halladas en los yacimientos costeros, también externo. Convencidos de esta premisa nos hacemos eco de las tempranas palabras de D. Potts "The growth of the Umm an-Nar culture in the second half of the third millennium may have delivered some of its energy from a lucrative arrangement with Sumer for trading copper, stone and wood" ${ }^{\prime 103}$. Sin embargo, ni las fuentes escritas ni los objetos mesopotámicos hallados en Omán nos hablan de la presencia de mercaderes sumeroacadios en el terreno; sí de redes comerciales muy bien trabadas desde hacía ya más de un milenio.

Los restos arqueológicos de Omán, en la fase que llamamos Umm an Nar, nos muestran una sociedad organizada a un nivel mayor que su antecesora Hafit, quizás esa diferencia permitió a los acadios mencionar la existencia de un rey; de un reino. Nosotros no podemos dar la razón a los textos acadios, pero sí mantener que esta sociedad contaba con artesanos especializados en el trabajo del metal y de la piedra, y con una economía mixta basada en la explotación de la palmera datilera, el cultivo de cereales, frutas, en la ganadería (en el interior) y en los recursos marinos (en la costa), utilizando el asno en sus desplazamientos por la península. Gracias al asno los kilos de cobre desplazados aumentarían con relación a la época Hafit, así como disminuiría el tiempo que se necesitaría en llevarlo desde el interior a ambas costas de la Península. El rastro de la transformación del cobre desde las zonas de extracción a

\footnotetext{
${ }^{99}$ S. CLEUZIOU y M. TOSI, 2007, pp. 93-95

${ }^{100}$ P. MAGEE, 2014, p. 96

${ }^{101}$ P. MAGEE, 2014, p. 105. El autor no duda de la importancia de este animal en la creación de redes comerciales intra-regionales, "The domesticated ass could move people and goods across the landscape in a fashion hitherto unknown in south-eastern Arabia. There can be little doubt that it was critical for the emergence of intraregional trade networks that engaged in growing regional maritime trade”, p. 106.

${ }^{102}$ A. HASTINGS, J.H. HUMPHRIES y R.H. MEADOW, “Oman in the Third Millennium BCE” JOS 1, 1975, p. 12.

${ }^{103}$ D.T. POTTS, "Towards an Integrated History of Culture Change in the Arabian Gulf Area: Notes on Dilmun, Makkan and the Economy of Ancient Sumer" JOS 4, 1978, p. 45.
} 
los oasis del piedemonte de las Montañas de al Hayyar y de allí a los asentamientos costeros, permite asegurar un trasvase interno del metal, que se limpia de sus mayores impurezas en las zonas mineras, donde además se transforma en lingotes, y que se vuelve a limpiar en los asentamientos costeros y del interior, donde se trasforma en armas y utensilios. Los objetos acabados son destinados a las magníficas tumbas Umm an Nar o a los asentamientos locales, pero no se enviarían a Mesopotamia, donde el alto grado de especialización de los artesanos acadios y una mentalidad diferente, les haría permanecer a la espera de los lingotes o del material en bruto para modelarlo a su gusto, ya que los objetos en cobre y en bronce hallados en los niveles acadios de los yacimientos mesopotámicos así lo indica.

Pero Omán, con ello, ya se ha abierto al exterior y busca en las montañas y en la tierra que cultiva, no sólo su propio abastecimiento, sino un excedente que envía a través del Golfo a Mesopotamia, excedente de cobre y de "piedra negra", sin embargo, los textos mencionan también dátil su $\mathbf{s u}_{11}$-lum / suluppu, caña še / uțtatu y madera ĝ ǧš / iṣu, que denominan "madera de Magan". De todos ellos, al menos el cobre nos permite asegurar la existencia de un grupo de personas amplio, dedicado a su explotación y transformación si tenemos en cuenta que los investigadores calculan que en la segunda mitad del III milenio a.C. se manejaron entre 2.000 y 4.000 toneladas de cobre en Omán, un nivel de producción que no ha sido hallado en ninguna otra parte del Próximo Oriente ${ }^{104}$, y que solo en el Wādi al-Hilo, donde una pequeña área de trabajo se asocia a un pequeño asentamiento se calculan 28 toneladas.

No podemos dudar a tenor de los hallazgos en la Península, que esta región del Golfo participa de lleno en los circuitos comerciales del Próximo Oriente, y no solo sus materias primas sino el eco de la existencia de una sociedad organizada, que introduce esos bienes en esa red comercial, tuvo que llegar forzosamente a Mesopotamia desde finales del Dinástico Temprano. Pero la mentalidad en Mesopotamia había cambiado, la ideología acadia de la expansión y toma directa de productos para la creación de un gran reino y una ciudad digna de semejante estado, tuvo que imprimir forzosamente la salida de sus reyes fuera del territorio mesopotámico, como ha quedado demostrado en otras regiones circundantes. Que los reyes acadios "cruzaran el Mar Inferior", pisaran las montañas de Omán, recorrieran sus wadiān, vieran por sí mismos extraer los metales de sus minas e impusieran sus condiciones al "rey" omaní es algo que no podemos asegurar, probablemente no fue así. Pero que las dos entidades, la cultura Umm an Nar y el reino acadio se transmitieron materiales, objetos e ideas no nos queda duda, ambas se reconocieron y se observaron, la primera atesorando cerámica acadia en sus tumbas y la segunda demandando al menos cobre omaní, de cuya presencia en los circuitos comerciales que unen el Golfo Pérsico con el resto del Próximo Oriente ya no podemos dudar.

${ }^{104}$ L.R. WEEKS, 1997, p. 16. 


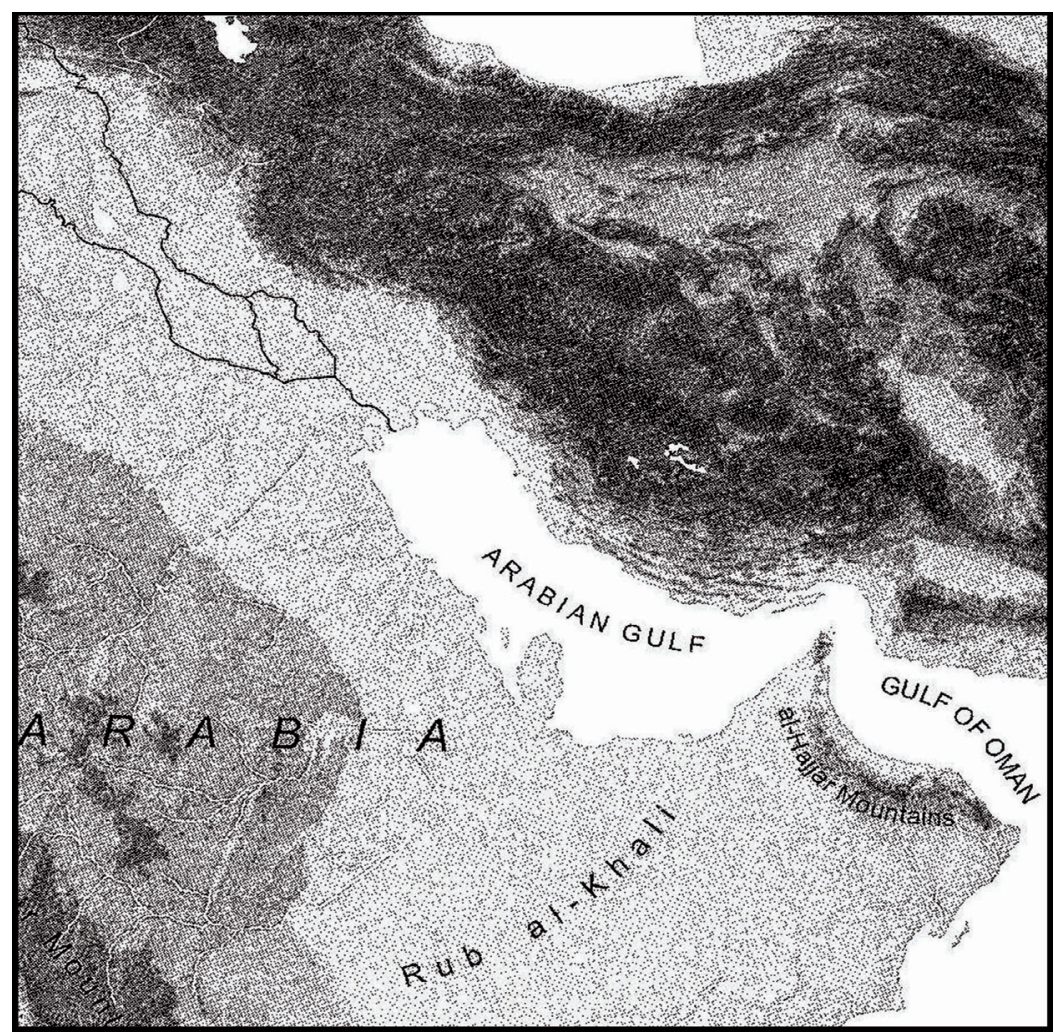

Fig. 1. Mapa físico de Mesopotamia y el Golfo Pérsico, P. MAGEE, 2014, p. 15

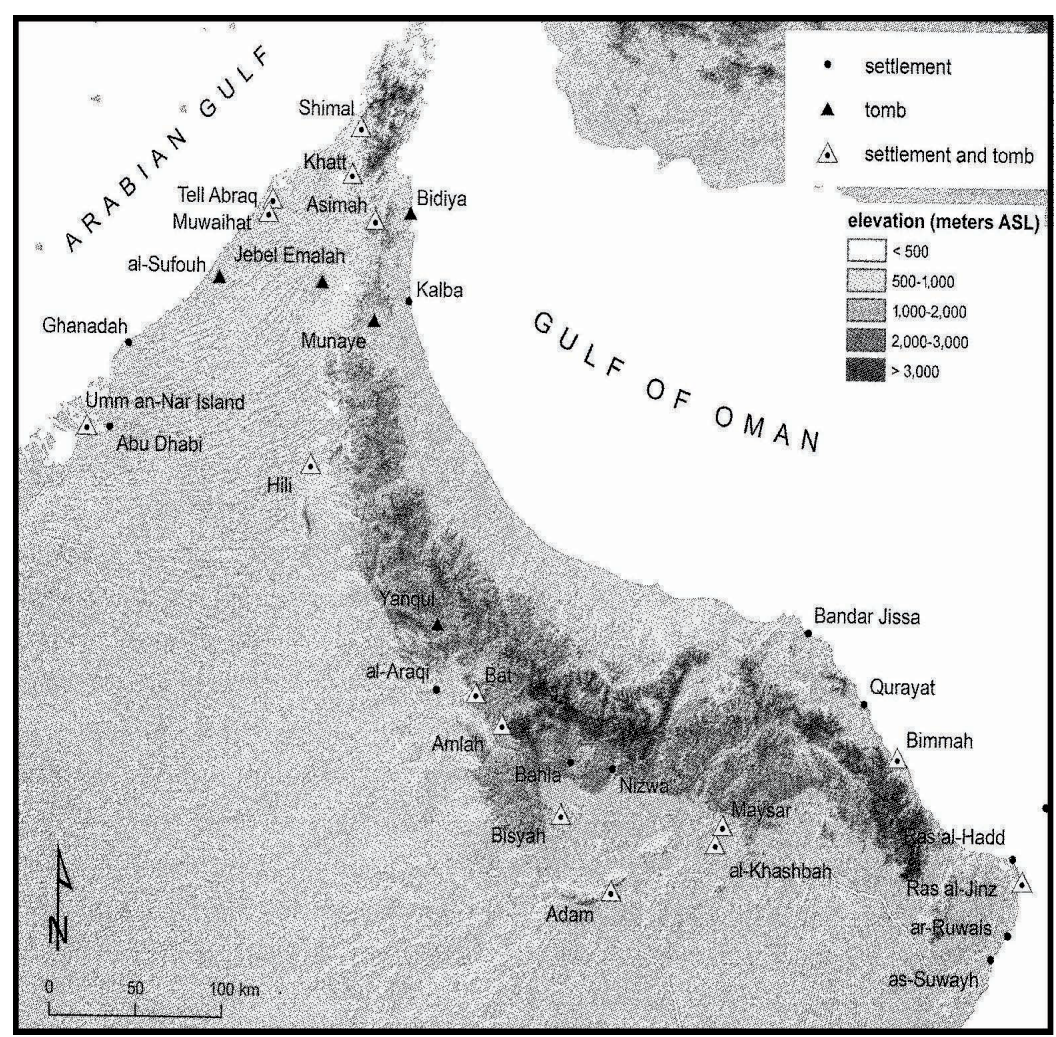

Fig. 2. Mapa de la Península de Omán con los asentamientos de época Umm an Nar, P. MAGEE, 2014, p. 99 


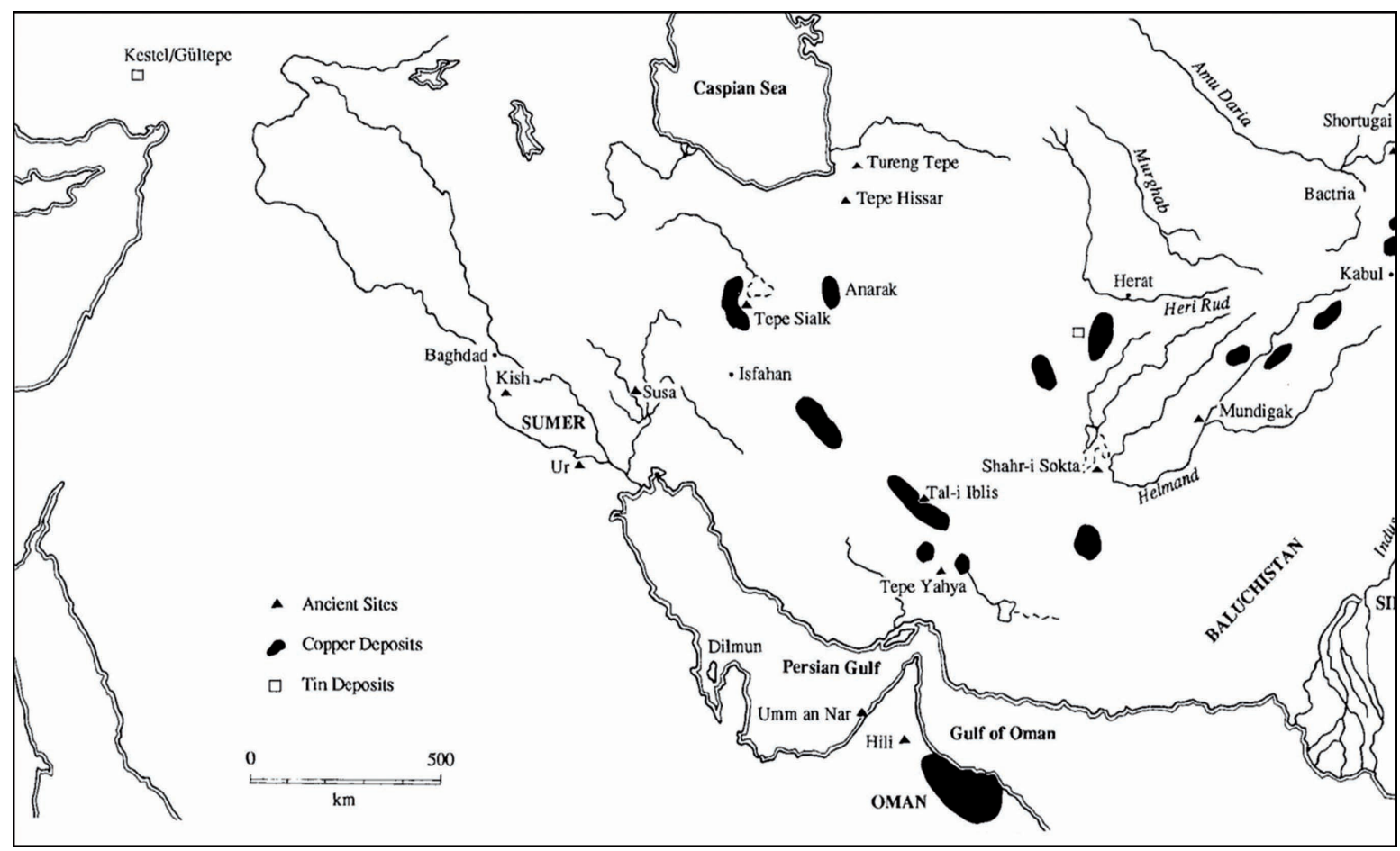

Fig. 3. Mapa con los depósitos más importantes de cobre y estaño. D.T. POTTS, 1994a, p. 226

\section{BIBLIOGRAFÍA}

ABDUL-HADI AL-FOUADI

1976 "Bassetki Statue with Old Akkadian Inscription of Naram-Sin of Agade (B.C. 2291-2255)", Sumer 32, pp. 63-75.

\section{BEECH, M.}

2003 “Archaeobotanical evidence for Early Date consumption in the Arabian Gulf', en J. S. al Swaidi (dir.), The Date Palm. From traditional Resource to Green Wealth, Abu Dhabi, pp. 11-32.

BEGEMANN, F. ET AL.

2010 "Lead isotope and chemical signature of copper from Oman and its occurrence in Mesopotamia and sites on the Arabian Gulf coast", AAE 21, pp. 135-169.

BOISSIER, A.

1919 “Inscription de Narâm-Sin”, $R A$ 16, pp. 157-164.

del CERRO, C.

2009 "La península de Omán: sociedad y usos del entorno en los oasis de la antigua Magan", Isimu 10, pp. 239-254.

2013 "Gran rey, rey del mundo, rey de Asiria... ¿Rey de reyes de las tierras de Dilmun, Magan y Meluhha?", Isimu 16, pp. 47-64.

CLEUZIOU, S.

1977-79 Archeologie aux Emirats Arabs Unis. Vol I-III, Al-Ain.

1980 "Économie et société de la Penínsule d'Oman au III" millénaire; le rôle des analogies interculturelles", Colloques internationaux du CNRS 580, pp. 342-359. 
1989a "Excavations at Hili 8; a preliminary report on the 4th and 7th campaigns", Archaeology in the United Arab Emirates V, pp. 61-87 y figs. 9-35a.

$1989 \mathrm{~b}$ "The chronology of protohistoric Oman as seen from Hili", en P. Costa y M. Tosi (eds.), Oman Studies. Serie Orientale Roma 63, Roma, pp. 47-78.

1998 "Enjeux et Perspectives de la Recherche Archéologique dans la Péninsule Arabique, de la Fin de la Préhistoire à l'Âge du Bronze", Isimu 1, pp. 43-62.

CLEUZIOU S. y MÉRY S.

2002 "In between the great Powers in the Bronze Age Oman Peninsula", en S. Cleuziou et al. (eds.), Essays on the late Prehistory of the Arabian Peninsula, Serie Orientale Roma 93, Roma, pp. 273-316.

CLEUZIOU, S., MÉRY, S. y VOGT, B.

2011 Protohistorie de l'oasis d'Al-Ain, Travaux de la Mission archéologique française à Abou Dhabi (Emirats Arabes Unis). BAR International Series 2227, Oxford.

CLEUZIOU S. y TOSI M.

2007 In the shadow of ancestors. The prehistoric foundations of the early Arabian civilization in Oman, Muscat.

CÓRDOBA, J.Ma . (Coord.)

2016 En los confines del Oriente Próximo El hallazgo moderno del país de Magán Veinte años de descubrimientos del Departamento de Antigüedades de Sharjah, la comunidad cientifica internacional y la misión de la Universidad Autónoma de Madrid en Sharjah (Emiratos Árabes Unidos), Madrid.

COSTA, P.M. y WILKINSON, T.J.

1987a "Settlement and Copper Exploitation in the 'Arja area", JOS 9, pp. 93-131.

$1987 b$ “The Environmental Setting of the Sohar Region”, JOS 9, pp. 23-33.

CRAWFORD, H. E. W.

1973 "Mesopotamia's Invisible Exports in the Third Millennium B.C.", World Archaeology 5/2, pp. 232-241.

EDZARD, D.O., FARBER G. y SOLLBERGER, E.

1977 Répertoire Géographique des Textes Cunéiformes I. TAVO Reihe 13, 7/1, Wiesbaden.

ENGLUND, R.

1983 "Dilmun in the Archaic Uruk Corpus", BBVO 2, pp. 35-37.

FAISAL BIN ALI AL SAID

1987 “The Environmental Setting of the Sohar Region”, JOS 9, pp. 30-33.

FRAYNE, D.

1993 The royal Inscriptions of Mesopotamia early periods, volume 2. Sargonic and Gutian periods (2334-2113 BC), London. 
FRIFELT, K.

1975a "A possible link between Jemdet Nasr and the Umm an-Nar graves of Oman", JOS 1, pp. 57-80.

1975b "On Prehistoric Settlement and Chronology of the Oman Peninsula" East and. West 25, pp. 359-424.

1991 The island of Umm an-Nar, vol I: third millennium graves. Jutland Archaeological Society Publications, 26/1, Copenhagen.

1995 The island of Umm an-Nar, vol II: the third millennium settlement. Jutland Archaeological Society Publications, 26/2, Copenhagen.

FOSTER B.R.

1977 "Commercial activity in sargonic Mesopotamia”, Iraq 39, pp. 31-43.

1990 "Naram-Sin in Martu and Magan", ARRIM 8, pp. 25-44.

2016 The Age of Agade Inventing Empire in ancient Mesopotamia, Oxon-New York.

GLASSNER, J.J.

1989 "Mesopotamian textual evidence on Magan/Makan in the late 3rd Millennium BC", en P. Costa y M, Tosi (ed.), Oman studies. Papers on the archaeology and history of Oman, Serie Orientale Roma 63, pp. 181-191.

GRAYSON, A.K.

2000 Assyrian and Babylonian Chronicles, Winona Lake.

GLEB I.J. y KIENAST, B.

1990 Die Altakkdischen Königsinschriften der Dritten Jahrtausends Vor. Chr., FAOS 7, Stuttgart.

GOODENOUGH, K. M. ET AL.

2014 "Records of Ocean Growth and Destruction in the Oman-UAE Ophiolite", Elements 10, pp. 109-114.

HASTINGS, A., HUMPHRIES J.H. y MEADOW, R.H.

1975 "Oman in the Third Millennium BCE”, JOS 1, pp. 9-55.

HAUPTMANN, A.

19855000 Jahre Kupfer in Oman. Band 1: Die Entwicklung der Kupfermetallurgie vom 3. Jahrtausend bis zur Neuzeit Der Anschnitt, Beiheft 4, Bochum.

HAUPTMANN A. y WEISGERBER G.

1981 “Third millenium BC copper production in Oman”, Revue d'Archéométrie 1, pp. 131-138.

HEIMPEL W.

1982 “A first step in the diorite question", $R A$ 76, pp. 67-67.

HUMPHRIES, J.H.

1974 "Harvard Archaeological survey in Oman: II- Some later prehistoric sites in the Sultanate de Oman", PSAS 4, pp. 49-77. 
KUTTERER J. y JASIM S.A.

2009 "First report on the copper-smelting site HLO-1 in Wādī al-Hilo, UAE", PSAS 39 , pp. 245-254.

JASIM S.A. y YOUSIF E.

2016 "Bronze Age Tombs from Jebel Faya, Emirate of Sharjah", Sharjah Archaeology 15 , pp. 8-17.

JASIM, S.A., UERPMANN M. y. UERPMANN, H.P.

2016 Mleiha: the unwritten History. Sharjah.

MAGEE, P.

2014 The Archaeology of Arabian Peninsula. Adaptation and Social Formation from Neolithic to the Iron Age, Cambridge.

MAGEE, P. ET AL.

2015 "Report on Excavations at Tell Abraq, Emirate of Sharjah, United Arab Emirates 2010-2013”, Sharjah Antiquities 14, pp. 5-29.

MAÑ́, $M$.

2005 La arqueología de la muerte en la península de Oman (III mil. a.C.) Prácticas y creencias en la región del Piedemonte, Supplementa ad Isimu. I Series: Sudia vol 3, Madrid.

MÉRY, S.

1991 "Origine et production des récipients de terre cuite dans la péninsule d'Oman à l'Âge du Bronze", Paléorient 17, pp. 51-78.

2000 "La céramique Mésopotamienne de Période Hafit" en S. Méry (ed.), Les céramiques d'Oman et l'Asie moyenne. Une archéologie des échanges à l'Âge du Bronze. Monographies du CNRS 23, Paris, pp. 170-189.

MÉRY, S. y TENGBERG, M.

2009 "Food for eternity? The analysis of a date offering from a 3rd Millennium BC grave at Hili N, Abu Dhabi (United Arab Emirates)", Journal of Archaeological Science 36, pp. 2012-2017.

MOOREY, P.R.S.

1982 "The archaeological evidence for metallurgy and related technologies in Mesopotamia” Iraq 44, pp. 13-38.

MUHAMMED ABDUL NAYEEM

1994 The United Arab Emirates, Hyderabad.

NYLANDER, C.

1980 “Earless in Nineveh: Who Mutilated "Sargon's" Head?” AJA 84/3, pp. 329-333.

PEAKE, $\mathrm{H}$.

1928 “The Copper Mountain of Magan”, Antiquity 2, pp. 452-457. 
PETTINATO, G.

1972 "El commercio con l'estero della Mesopotamia meridionale nel 3 millenio av. Cr, alla luce delle fonti letterarie e lessicali sumeriche", Mesopotamia 7, pp. 43-166.

\section{POTTS D.T.}

1978 "Towards an Integrated History of Culture Change in the Arabian Gulf Area: Notes on Dilmun, Makkan and the Economy of Ancient Sumer", JOS 4, pp. 29-51.

1986 "Eastern Arabia and the Oman peninsula during the late fourth and early third millennium BC", en U. Finkbeiner y W. Röllig (eds.), Gamdat Nasr: Period or Regional Style?, Wiesbaden, pp. 121-170.

1992a "The chronology of archaeological assemblages from the head of the Arabian Gulf to the Arabian Sea (8000-1750 BC)", en R. W. Ehrich (ed.), Chronologies in Old World Archaeology, Chapter IV, Chicago, pp. 63-89.

1992b The Arabian Gulf in Antiquity. Vol 1, Oxford.

1993a "The Late Prehistoric, Protohistoric, and Early Historic Periods in Eastern Arabia (ca. 5000-1200 B.C.)", Journal of World Prehistory 7/2, pp. 163-212.

1993b "Four seasons of excavation at Tell Abraq (1989-1993)", PSAS 23, pp. 117-126.

1994a Mesopotamian and the east. An Archaeological and Historical Study of Foreign relations 3400-2000 BC, Oxford.

1994b The Arabian Gulf in Antiquity. Vol I. From Prehistory to the Fall of the Achaemenid Empire, Oxford.

$1994 \mathrm{c}$ "Contributions to the agrarian history of Eastern Arabia II. The cultivar", $A A E$ 5, pp. 236-275.

2000 Ancient Magan, The Secrets of Tell Abraq. London-Abu Dhabi.

2003 'Date palms and date consumption in Eastern Arabia during the Bronze Age', en J.S. al Swaidi (dir.), The Date Palm. From traditional Resource to Green Wealth, Abu Dhabi, pp. 33-50.

RICE, M.

1994 The archaeology of the Arabian Gulf. 5000-323 B.C., London.

SOLLBERGER, E.

1971, Inscriptions Royales Sumeriennes et Akkadiennes, Paris.

AL TIKRITI, W.Y.

2001 Archaeology of Umm an-Nar island: 1959-2009, Abu Dhabi.

THUREAU-DAGIN, F. ET AL.

1910 Inventaire des tablettes de Tello conservées au Musée Impérial Ottoman (ITT) I, Paris.

1912 Inventaire des tablettes de Tello conservées au Musée Impérial Ottoman (ITT) II, Paris.

\section{TOSI, M.}

1976 "Notes on the Distribution and Exploitation of Natural Resources in Ancient Oman”, JOS 1, pp. 187-206.

UERPMANN M. y UERPMANN, H.P.

2008 "Animal economy during the Early Bronze Age in South-East Arabia" en E. Vila et al. (eds.), Archaeozoology of the Near East 8, TMO, 49, Lyon, pp. 146-185. 
VOGT, B.

1985 "The Umm an Nar Tomb A at Hili North: a preliminary report on three seasons of excavation, 1982-1984”, Archaeology of Unites Arab Emirates 4, pp. 20-37.

WEEKS, L.R.

1997 "Prehistoric Metallurgy at Tell Abraq, U.A.E", AAE 8, pp. 11-85.

2003 Pre-Islamic Metallurgy of the Gulf, Technology, trade and the Bronze Age World. American School of Prehistoric Research Monograph series, Boston/Leiden.

WEISGERBER, G.

1978 "Evidence of Ancient Mining Sites in Oman: a Preliminary Report", JOS 4, pp. 15-28, Taf. 11a-26.

1987 “Archaeological Evidence of Copper Exploitation at 'Arja”, JOS 9, pp. 145-172.

WEISGERBER, G. y YULE, P.

2003 'Al-Aqir near Bahlā' an Early Bronze Age dam site with planoconvex 'copper' ingots", $A A E$ 14, pp. 24-53.

WESTENHOLZ J.G.

1997 Legends of the Kings of Akkade. The Texts, Winona Lake.

YULE P., y GUBA, I.

2001 "Did the Ancient Mesopotamian Royal Stone Originate in Oman?", Adumatu, 4 pp. 41-52.

ZIOLKOWSKI, M.C.

2001 "The Soft Stone Vessels from Sharm, Fujeirah, United Arab Emirates", AAE 12, pp. 25-30. 